\title{
Relationship between Type 2 Diabetes Mellitus and the occurrence of Gastroesophageal varices in patients with chronic Hepatitis C Virus Related Liver Cirrhosis \\ Fathy G. Abdel-Raziq, Mahmoud S. Berengy, Tarek M. Emran ${ }^{(*)}$, Ahmed RA Mohammed \\ Internal Medicine, and Clinical Pathology (*) Departments, Faculty of Medicine, Al-Azhar University (Damietta)
}

\begin{abstract}
Background: Hepatitis C virus (HCV) infection is a challenging health problem in Egypt. Esophageal varices are a major complication of it which may bleed and endanger patient's life.

Aim of the work: to assess the relationship between type-2 DM and the development of gastroesophageal varices and explore the role of insulin resistance as a predictor of gastroesophageal varices.

Patients and methods: This study included 100 patients with Child A, HCV-induced cirrhosis. They were divided into two main groups: Group A included 50 patients with type-2 DM, while Group B: included 50 non-diabetic which were subdivided into: Group B1: patients without DM but, with insulin resistance (IR) $\{32\}$, and Group B2: patients without DM or IR $\{18\}$. All patients were subjected to full history taking, clinical examination, laboratory and imaging studies (abdominal ultrasound) and upper GI endoscopy.

Results: The prevalence of esophageal varices in patients with Child A HCV-induced cirrhosis was $80 \%$, elevated to $88 \%$ in patients with type- 2 DM. Insulin resistance played the major role in development of esophageal varices. There are statistically significant elevated HOMA-IR score, lower platelet count/spleen diameter ratio and higher right liver lobe diameter/albumin ratio in patients with varices.

Conclusion: Insulin resistance is a major contributor for development of esophageal varices in HCV induced cirrhosis. Platelet count/spleen diameter ratio, right liver lobe diameter/albumin ratio and insulin resistance measured by HOMA-IR are good predictors for the presence of esophageal varices. Keywords: type-2 diabetes mellitus, insulin resistance, hepatitis $\mathrm{C}$ virus infection, gastroesophageal
\end{abstract} varices.

\section{Introduction}

Hepatitis $\mathrm{C}$ virus (HCV) infection is a global health problem, which can lead to progressive hepatic injury with consequent cirrhosis and end stage liver disease ${ }^{[1]}$. Worldwide, Egypt is an endemic country with the highest prevalence of $\mathrm{HCV}$ infection $(15 \%)^{[2]}$. Gastroesophageal varices (GEV) are serious complication of portal hypertension, with variceal bleeding reported in up to $50 \%$ of cirrhotic patients. The mortality of bleeding is up to $20 \%$ irrespective of improved diagnostic and therapy modalities. Variceal bleeding is the second most common cause of mortality in patients with cirrhosis ${ }^{[3]}$.

About two thirds of patients with decompensated cirrhosis and one third of patients with compensated cirrhosis have GEV at the time of diagnosis. Thus, it is very important to recognize and treat patients at highest risk ${ }^{[4]}$. Cherian et al ${ }^{[5]}$ proposed that screening of all cirrhotic patients with an endoscopy is mandatory to detect GEV and to initiate prophylactic maneuvers in patients with large GEV. Abu El Makarem et al. ${ }^{[6]}$ suggested that non-invasive prediction of GEV is in great demand to prevent unnecessary endoscopy and the unnecessary cost. This cost is of concern in many African countries, including Egypt, where liver cirrhosis is highly prevalent. On the other side, Berzigotti et al..$^{[7]}$ proposed the criteria of ideal method for recognition of varices. It must be simple, noninvasive, cheap, reproducible, precise, and readily accessible; have high sensitivity and specificity; follow the natural history; detect the effect of the treatment correctly; and indicate the prognosis and possibility of treatment.

Type-2 diabetes mellitus (type-2 DM) comprised patients with insulin resistance (IR) and relative insulin deficiency ${ }^{[8]}$. IR was linked to HCV infection and usually developed early in the course of the disease ${ }^{[9]}$. Thus, IR is proposed to have a possible usefulness in prediction of GEV in patients with early cirrhosis. It is simple, non-invasive, and easyto-get test ${ }^{[10]}$. In addition, IR was reported as a major independent determinant of fibrosis and 
chronicity in HCV chronic infection. IR was not affected by HCV genotype or liver damage severity ${ }^{[11]}$. Also, Hung et al..$^{[12]}$ reported that patients infected with HCV have significantly higher IR than healthy controls matched for age, sex and body mass index (BMI). Esmat $\boldsymbol{e t}$ al..$^{[13]}$ suggested that platelet count/spleen diameter ratio and right liver lobe diameter/albumin concentration ratio can be used also as non-invasive predictors for GEV.

The aim of this study is to assess the relationship between type-2 DM and IR from one side and the development of GEV on the other side. In addition, to evaluate the role of IR as independent risk factor and predictor of GEV. Finally, if we could consider platelet count/spleen diameter ratio and right liver lobe diameter/albumin ratio as reliable predictors for the existence of GEV in patients with compensated HCV induced cirrhosis.

\section{Patients and methods}

This study was carried out in the Internal Medicine Department and the Clinical Pathology Department, Faculty of Medicine, Al-Azhar University (New Damietta) during the period from February 2018 to September 2018. This study included 100 patients with Child A, HCV-induced cirrhosis (60 male and 40 females with age range from 30 to 70 years). All patients provided an informed consent before participating in this study.

According to the presence or absence of type-2 DM, the studied patients were divided into two main groups. Group $A$ included 50 patients with type-2 DM, while Group B: included 50 patients without DM. Then, according to the presence or absence of insulin resistance (IR), the non- diabetic patients were further subdivided into two subgroups: Group B1: patients without DM but, with insulin resistance (IR) $\{32\}$, and Group B2: patients without DM or IR $\{18\}$. Patients were included as they had a diagnosis of $\mathrm{HCV}$-induced cirrhosis based on clinical, laboratory and imaging criteria. On the other side, patients who had any of the following: advanced cirrhosis (Child classes B and C), history of upper GIT bleeding, hepatic or extrahepatic malignancies, portal vein thrombosis, Budd Chiari syndrome, advanced cardiac or renal disease, isolated gastric varices, bilharzial periportal fibrosis, current treatment with beta-blockers, diuretics, or other drugs affecting portal blood pressure, type-1 DM, past or present treatment by antiviral drugs were excluded.

All patients of the study were subjected to full history taking and clinical examination. Body mass index (BMI) was calculated as weight in kilograms/height in square meters and patients were assigned as normal, overweight, and obese according to classification of Flegal et al. ${ }^{[14]}$. A blood sample was taken and the following laboratory investigations were done: complete blood count (CBC), liver function tests (bilirubin, albumin, SGPT, SGOT), renal function tests (serum creatinine), coagulation profile (PT and INR), fasting and postprandial plasma glucose levels, viral markers for HCV and HBV (kits supplied by Abon Biopharm (Hangzhou) Co., Ltd), fasting insulin assay (using kits provided by Monobind USA (AccuBind ELISA Microwells). Finally, IR was calculated by the homeostasis model assessment (HOMA) method using the following equation:(HOMAIR $)=$ fasting insulin $(\mu \mathrm{U} / \mathrm{ml}) \times$ fasting glucose $(\mathrm{mg} / \mathrm{dl}) / 405$ (normal $<2.6)^{[15]}$.

The Child-Pugh score was calculated by the methods described by Pugh et al. ${ }^{[16]}$.A total score of 5-6 was considered stage A (well-compensated disease); 7-9 was stage B (significant functional compromise); and 1015 was stage $\mathrm{C}$ (decompensated disease). A pelvi-abdominal ultrasonography examination was performed to evaluate findings that suggest cirrhosis (irregular border, coarse surface and attenuated blood supply), and to measure the portal vein diameter (normally up to $13 \mathrm{~mm}$ ) and longitudinal (bipolar) diameter of the spleen (normally about $11 \mathrm{~cm}$ ). In addition, to measure right liver lobe diameter in mid-clavicular line (normally about $12 \mathrm{~cm}$ ). The examination was carried out by (TOSHIBA Aplio 500®) system. All patients underwent an upper gastrointestinal endoscopy. All endoscopies were performed in the endoscopy unit by an experienced endoscopist using a flexible video gastroscope (Olympus Medical Systems, Japan). Esophageal varices were graded as by Alempijevic et al.$^{[17]}$ into four grades. Finally, calculation of the right liver lobe diameter $(\mathrm{cm}) /$ serum albumin concentration $(\mathrm{gm} / \mathrm{dl})$, and platelet count/spleen bipolar diameter (mm) were carried out.

Statistical Analysis: All statistical calculations were performed using computer programs Microsoft Excel 2007 (Microsoft Corporation, WA, USA) and SPSS (Statistical 
Package for the Social Science; SPSS Inc., Chicago, IL, USA) version 22 for Microsoft Windows. Quantitative data expressed as mean \pm standard deviation (SD), data was analyzed by independent sample $t$ test. While qualitative data were expressed as number and percentage and were analyzed by Chi square $\left(\mathrm{X}^{2}\right)$ test. The receiver operating characteristic (ROC) curve was performed to determine cut-off values for the studied technique. Sensitivity and specificity were determined. The threshold of significance was fixed at 5\% level ( $\mathrm{P}$ value). $\mathrm{P}$ value was considered significant if $<0.05$. The smaller the $\mathrm{P}$ value obtained the more significant are the results.

\section{Results}

There is statistically significant increase of EV in Group A (88.0\%) when compared to Group B (72.0\%). In addition, there was significant increase of EV in group A and B1 (non-diabetics with IR; 87.5\%) when compared to B2 group (non-diabetics without IR; 44.4\%). There is statistically significant increase in BMI in group A in comparison to group B or B2 and in group B1 when compared to $B 2$, and statistically significant increase in the presence of hypertension and family history of DM in group $\mathrm{A}$ in comparison to group B, B1 or B2 (Table 1).

There was statistically significant decrease in platelet count and significant increase in SGPT, SGOT, serum total bilirubin, fasting blood glucose, two hours postprandial blood glucose, $\mathrm{Hb} \mathrm{A} 1 \mathrm{c}$, fasting plasma insulin, HOMA-IR, serum cholesterol and serum triglycerides of group $\mathrm{A}$ in comparison to group B. In addition, there was significant decrease of liver enzymes, fasting, two hours post prandial blood sugar, HA1c, fasting plasma insulin, HOMA-IR, serum cholesterol and triglycerides in group B1 when compared to group A. Furthermore, there was statistically significant increase of hemoglobin, platelets and albumin and significant decrease of INR, liver enzymes, bilirubin, fasting and PP blood glucose, fasting insulin, HOMA-IR, serum cholesterol and triglycerides in group B2 when compared to group A. In addition, there was statistically significant decrease in platelet count and significant increase in INR, SGPT, SGOT, serum albumin, serum total bilirubin, fasting blood glucose, two hours postprandial blood glucose, $\mathrm{Hb} \mathrm{A} 1 \mathrm{c}$, fasting plasma insulin and HOMA-IR in Group B1 in comparison to group B2 (Table 2).

There was statistically significant increase in the diameter of the portal vein $(\mathrm{p}=$ $0.011)$ and bipolar diameter of the spleen $(\mathrm{p}=$ $0.037)$ and significant decrease in PLT count $(\mathrm{n} / \mathrm{ul}) /$ spleen diameter $(\mathrm{mm})$ ratio $(\mathrm{p}=0.038)$ of group A in comparison to group B. There was significant increase of right lobe of the liver diameter and platelet count/spleen diameter ratio in $\mathrm{B} 2$ when compared to group $A$ and significant decrease of portal vein diameter, bipolar diameter of the spleen and right liver lobe/albumin ratio in B2 when compared to group A. Furthermore, there was statistically significant decrease in the right lobe of the liver diameter and platelet count/spleen diameter ratio and significant increase in the diameter of the portal vein, bipolar diameter of the spleen and right liver lobe/albumin ratio in B1 in comparison to B2 group (Table 3).

On comparing patients with esophageal varices to those without varices, there was significant increase of BMI $(29.09 \pm 2.33$ vs $27.14 \pm 3.27)$, family history of DM (58.8\% vs $35.0 \%$ ), diabetes $(55.0 \%$ vs $30.0 \%)$, presence of IR $(90.0 \%$ vs $50.0 \%)$, INR $(1.35 \pm 0.166$ vs $1.04 \pm 0.05)$, bilirubin $\quad(1.51 \pm 0.22 \quad$ vs $1.02 \pm 0.08)$, fasting blood glucose $(156.79 \pm 66.80$ vs $122.30 \pm 64.50)$, fasting plasma insulin $(26.92 \pm 14.26$ vs $16.30 \pm 14.11)$, HOMA IR $(26.92 \pm 14.26$ vs $16.30 \pm 14.11)$. However, there was significant decrease of HB $(11.24 \pm 1.37$ vs $6.89 \pm 8.81)$, platelets $(112.665 \pm 24.29$ vs $166.50 \pm 9.26)$, albumin $(3.43 \pm 0.23$ vs $3.98 \pm 0.12)$ in $\mathrm{EV}$ when compared to no EV.

There was statistically significant decrease in the diameter of the right lobe of the liver ( $\mathrm{p}<0.001)$ and PLT count ( $\mathrm{n} / \mathrm{ul})$ / spleen diameter $(\mathrm{mm})$ ratio $(\mathrm{p}<0.001)$ and significant increase in the diameter of the portal vein $(\mathrm{p}$ $<0.001$ ), bipolar diameter of the spleen ( $\mathrm{p}$ $<0.001)$ and right liver lobe $(\mathrm{cm}) /$ albumin $(\mathrm{gm} / \mathrm{dl})$ ratio $(\mathrm{p}<0.001)$ of patients with $\mathrm{EV}$ in comparison to patients without EV (Table 4).

Receiver operating characteristic (ROC) curve analysis identified a HOMA-IR score of greater than 4.80 [sensitivity $=70 \%$ and specificity $=60 \%$ ] as the best cut-off for predicting the presence of EV in both diabetic and non-diabetic groups. In non-diabetic patients, a HOMA-IR score of greater than 2.16 [sensitivity $=80.6 \%$ and specificity $=$ $71.4 \%$ ] as the best cut-off for predicting the 
Fathy Abdel-Raziq et al.

presence of EV in group B. A PLT count (n/ $\mathrm{ul})$ / spleen diameter $(\mathrm{mm})$ ratio of lower than 1105.93 [sensitivity $=100 \%$ and specificity $=$ 98.8\%] is identified as the best cut-off for predicting the presence of $\mathrm{EV}$, while a right liver lobe/albumin ratio of greater than 4.133 [sensitivity $=93.8 \%$ and specificity $=90 \%$ ] was the best cut-off for predicting the presence of EV (Table 5).

Table (1): Comparison between studied groups as regard EV, demographics and risk factors

\begin{tabular}{|c|c|c|c|c|c|c|}
\hline \multicolumn{2}{|c|}{ Variables } & Group A & Group B & B1 & B2 & A vs B \\
\hline \multicolumn{2}{|c|}{ Esophageal varices } & $44(88.0 \%)$ & $36(72.0 \%)$ & $28(87.5 \%)$ & $8(44.4 \%)^{\#}$ & $0.046^{*}$ \\
\hline \multicolumn{2}{|c|}{ Age (years) } & $49.48 \pm 8.52$ & $50.06 \pm 9.34$ & $49.78 \pm 8.43$ & $49.78 \pm 8.43$ & 0.74 \\
\hline \multicolumn{2}{|c|}{ BMI $\left(\mathrm{kg} / \mathrm{m}^{2}\right)$} & $29.24 \pm 1.93$ & $28.17 \pm 3.14$ & $29.10 \pm 2.92$ & $26.51 \pm 2.90^{\#}$ & $0.043^{*}$ \\
\hline \multirow{2}{*}{$\begin{array}{l}\text { Blood } \\
\text { Pressure }\end{array}$} & Systolic & $128.00 \pm 15.49$ & $129.00 \pm 166.29$ & $128.28 \pm 15.669$ & $130.28 \pm 17.70$ & 0.75 \\
\hline & Diastolic & $79.90 \pm 12.35$ & $79.40 \pm 10.53$ & $79.38 \pm 10.30$ & $79.44 \pm 11.23$ & 0.82 \\
\hline Sex & Male/female & $30 / 20$ & $30 / 20$ & $19 / 13$ & $11 / 7$ & 1.0 \\
\hline \multicolumn{2}{|c|}{ Hypertension } & $27(54.0 \%)$ & $17(34.0 \%)$ & $10(31.2)^{\#}$ & $7(38.9 \%)$ & $0.044 *$ \\
\hline \multicolumn{2}{|c|}{ Family history of DM } & $37(74.0 \%)$ & $17(34.0 \%)$ & $20(62.5 \%) \#$ & $5(27.8 \%) \#$ & $0.001 *$ \\
\hline
\end{tabular}

* indicate significance; \# significant decrease when compared to group A.

Table (2): Statistical analysis of laboratory data of group A in comparison to group B

\begin{tabular}{|l|c|c|c|c|c|}
\hline & Group A & Group B & B1 & B2 & A vs B \\
\hline $\mathrm{Hb}$ & $11.27 \pm 1.33$ & $11.66 \pm 1.45$ & $11.36 \pm 1.55$ & $12.18 \pm 1.12^{\$}$ & 0.173 \\
\hline WBCs & $6.89 \pm 2.61$ & $6.23 \pm 2.13$ & $6.08 \pm 2.34$ & $6.51 \pm 1.70$ & 0.173 \\
\hline Platelet & $117.20 \pm 30.31$ & $129.64 \pm 30.61$ & $120.97 \pm 28.73$ & $145.06 \pm 28.32^{\$}$ & $0.044^{*}$ \\
\hline INR & $1.31 \pm 0.193$ & $1.26 \pm 0.19$ & $1.31 \pm 0.18$ & $1.16 \pm 0.18^{\#}$ & 0.223 \\
\hline SGPT & $55.42 \pm 13.94$ & $44.10 \pm 14.49$ & $47.72 \pm 11.43^{\#}$ & $37.67 \pm 17.27^{\#}$ & $<0.001^{*}$ \\
\hline SGOT & $45.92 \pm 11.50$ & $37.94 \pm 13.02$ & $40.81 \pm 10.23^{\#}$ & $32.83 \pm 5.96^{\#}$ & $0.002^{*}$ \\
\hline Serum Albumin & $3.50 \pm 0.29$ & $3.58 \pm 0.31$ & $3.49 \pm 0.27$ & $3.75 \pm 0.31^{\$}$ & 0.195 \\
\hline Serum total Bilirubin & $1.474 \pm 0.26$ & $1.35 \pm 0.29$ & $1.43 \pm 0.27$ & $1.21 \pm 0.27^{\#}$ & $0.038^{*}$ \\
\hline Serum Creatinine & $1.25 \pm 0.53$ & $1.30 \pm 0.04$ & $1.35 \pm 0.58$ & $1.22 \pm 0.47$ & 0.628 \\
\hline Fasting blood glucose & $195.06 \pm 8.62$ & $104.72 \pm 17.99$ & $116.09 \pm 7.34^{\#}$ & $84.50 \pm 12.60^{\#}$ & $<0.001^{*}$ \\
\hline 2h p.p blood glucose & $246.98 \pm 13.04$ & $140.42 \pm 15.36$ & $144.56 \pm 17.26^{\#}$ & $133.06 \pm 6.86^{\#}$ & $<0.001^{*}$ \\
\hline Hb A1c & $8.04 \pm 1.27$ & $5.76 \pm 0.49$ & $5.91 \pm 0.33^{\#}$ & $5.49 \pm 0.60^{\#}$ & $<0.001^{*}$ \\
\hline Fasting plasma Insulin & $33.74 \pm 2.85$ & $15.84 \pm 1.65$ & $21.62 \pm 3.00^{\#}$ & $5.58 \pm 1.18^{\#}$ & $<0.001^{*}$ \\
\hline HOMA-IR & $18.29 \pm 1.81$ & $4.42 \pm 1.31$ & $6.23 \pm 1.77^{\#}$ & $1.20 \pm 0.38^{\#}$ & $<0.001^{*}$ \\
\hline Serum Cholesterol & $218.58 \pm 43.11$ & $169.14 \pm 31.68$ & $168.81 \pm 28.99^{\#}$ & $169.72 \pm 36.88^{\#}$ & $<0.001^{*}$ \\
\hline Serum Triglycerides & $182.34 \pm 5.62$ & $140.50 \pm 31.86$ & $141.34 \pm 29.82^{\#}$ & $139.00 \pm 36.05^{\#}$ & $<0.001^{*}$ \\
\hline
\end{tabular}

* indicate significance; \# significant decrease when compared to group A; \$ indicate significant increase when compared to group A.

Table (3): Abdominal ultrasonographic data, platelet count / spleen diameter ratio and right liver lobe/albumin) ratio of group A in comparison to group B

\begin{tabular}{|l|c|c|c|c|c|}
\hline & Group A & Group B & B1 & B2 & A vs B \\
\hline Diameter of the Right lobe of the liver & $152.90 \pm 3.872$ & $154.30 \pm 4.311$ & $153.16 \pm 3.95$ & $156.33 \pm 4.27^{\$}$ & 0.091 \\
\hline Diameter of the portal vein & $13.58 \pm 1.76$ & $12.62 \pm 1.92$ & $13.19 \pm 1.69$ & $11.61 \pm 1.91^{\#}$ & $0.011^{*}$ \\
\hline Bipolar diameter of the spleen & $147.92 \pm 9.60$ & $143.68 \pm 10.45$ & $145.59 \pm 9.71$ & $138.50 \pm 9.94^{\#}$ & $0.037^{*}$ \\
\hline PLT count/spleen diameter ratio & $808.73 \pm 62.12$ & $921.53 \pm 74.39$ & $840.99 \pm 50.6$ & $1064.69 \pm 261.86^{\$}$ & $0.038^{*}$ \\
\hline Right liver lobe/ Albumin ratio & $4.38 \pm 0.270$ & $4.32 \pm 0.27$ & $4.40 \pm 0.24$ & $4.19 \pm 0.27^{\#}$ & 0.294 \\
\hline
\end{tabular}

* indicate significance; \# significant decrease when compared to group A; \$ indicate significant increase when compared to group A.

Table (4): Comparison of abdominal ultrasonographic data and different ratios between patients with $\mathrm{EV}$ and those without $\mathrm{EV}$

\begin{tabular}{|l|c|c|c|c|l|}
\hline & \multicolumn{2}{|c|}{ EV (No.=80) } & \multicolumn{2}{|c|}{ No EV (No.=20) } & \multirow{2}{*}{ P } \\
\cline { 2 - 5 } & Mean & SD & Mean & SD & \\
\hline Diameter of the Right lobe of the liver $(\mathrm{mm})$ & 152.14 & 3.189 & 159.45 & 1.356 & $<0.001^{*}$ \\
\hline
\end{tabular}


Relationship between Type 2 Diabetes Mellitus and the occurrence of Gastroesophageal...

\begin{tabular}{|l|c|c|c|c|l|}
\hline Diameter of the portal vein (mm) & 13.84 & 1.27 & 10.15 & 0.75 & $<0.001^{*}$ \\
\hline Bipolar diameter of the spleen (mm) & 149.26 & 8.249 & 131.95 & 2.781 & $<0.001^{*}$ \\
\hline PLT count (n/ ul) / spleen diameter (mm) ratio & 765.512 & 22.062 & 1263.612 & 9.991 & $<0.001^{*}$ \\
\hline Right liver lobe (cm) / Albumin (gm/dl) ratio & 4.446 & 0.226 & 4.004 & 0.094 & $<0.001^{*}$ \\
\hline
\end{tabular}

Table (5): Sensitivity and specificity of factors associated with the presence of esophageal varices

\begin{tabular}{|l|c|c|c|c|c|}
\hline & Cut off & AUC & Sensitivity & Specificity & P value \\
\hline HOMA-IR (All patients) & 4.80 & 0.712 & $70 \%$ & $60 \%$ & $0.003^{*}$ \\
\hline HOMA-IR (group B) & 2.165 & 0.719 & $80.6 \%$ & $71.4 \%$ & $0.017^{*}$ \\
\hline PLT count/spleen diameter ratio & 1105.93 & 1.000 & $100 \%$ & $98.8 \%$ & $<0.001^{*}$ \\
\hline Right liver lobe/albumin ratio & 4.133 & 0.978 & $93.8 \%$ & $90 \%$ & $<0.001^{*}$ \\
\hline
\end{tabular}

Discussion

The aim of this study is to evaluate the relationship between type 2 diabetes mellitus and insulin resistance from one side and the occurrence of GEV on the other side, evaluate the role of insulin resistance as an independent risk factor and predictor of GEV and can we consider platelet count/spleen diameter ratio and right liver lobe diameter/albumin ratio reliable predictors for the presence of GEV in patients with compensated $\mathrm{HCV}$-induced cirrhosis attending at Al-Azhar University Hospital in New Damietta.

In the present study, there were statistically significant elevated fasting blood glucose levels, fasting plasma insulin levels and HOMA-IR score in HCV patients with EV than in those without $\mathrm{EV}$, these findings indicate a strong association between the presence of EV in HCV related liver cirrhosis and IR even in absence of diabetes. Insulin resistance is a risk factor for esophageal varices in cirrhotic patients with $\mathrm{HCV}$ infection. As the hepatic fibrosis is correlated with the development of EV, insulin resistance may be associated with the development of EV through progression of hepatic fibrosis ${ }^{[18]}$. Insulin modulates the endothelial synthesis of nitric oxide and endothelin, regulators of sinusoidal blood flow. Thus, insulin-induced hepatic fibrosis and vasoconstriction may be possible mechanisms for the development of $\mathrm{EV}^{[19]}$. Our findings agreed with Yosry $\boldsymbol{e t}$ $a l .{ }^{[20]}$ who stated that $83 \%$ of patients with chronic HCV infection and IR had esophageal varices.

In the present work, there were 32 from 50 patient $\{64 \%\}$ in the non-diabetic group (group B) had IR and only 18 patients $\{36 \%\}$ didn't have IR. Possible explanations of IR in chronic HCV (CHC) infection include direct viral effects on insulin signaling, contributions of inflammatory markers amplified by $\mathrm{CHC}$ and increased viral replication in hepatocytes. TNF- $\alpha$ induces IR by inhibition of insulin receptors and insulin receptor substrate (IRS)-1 phosphorylation, thus impairing insulin signaling ${ }^{[21]}$. Antuna et $a .^{[22]}$ reported that in patients with chronic HCV infection with IR and normal plasma glucose levels, the function of Beta cells was upgraded resulting in a statistically significant higher HOMA value compared with non-HCV patients. Our results agreed with Erice et al.$^{[23]}$ who reported that IR was found in $60 \%$ of patients with chronic $\mathrm{HCV}$ related liver cirrhosis.

In our study, the cut-off value for HOMA-IR score of greater than 4.8 was the optimal value for accurate prediction of EV with a resulting $70 \%$ sensitivity and $60 \%$ specificity in the whole study population and the cut-off value for HOMA-IR score of greater than 2.2 was the optimal value for accurate prediction of $\mathrm{EV}$ with a resulting $80.6 \%$ sensitivity and $71.4 \%$ specificity in the non-diabetic group (group B). Camma et al. ${ }^{[9]}$ found a significant association between HOMA-IR and EV in HCV related liver cirrhosis through his study which was conducted on 104 patients of Child A HCV induced cirrhosis and concluded that HOMAIR score of greater than 3.5 is the cut-off value with the sensitivity $61 \%$ and specificity $76 \%$ for predicting EV, he concluded that insulin resistance measured by HOMA-IR regardless of the presence of diabetes significantly predicts the presence of $\mathrm{EV}$ which is in agreement with our study. Wasfy et al..$^{[24]}$ found that at a cut off value equal to or more than 3.4, it could significantly predict EV with high sensitivity (75\%) and excellent specificity $(80 \%)$. The impact of IR in inducing EV in patients with HCV-related cirrhosis is more or less obvious in the study of Camma et al ${ }^{\left[{ }^{[9]}\right.}$ as 
well as the present study despite the different ethnic groups and different genotypes of $\mathrm{HCV}$ in studied groups.

In our study, there were statistically significant increase in weight, BMI and family history of DM in patients with $\mathrm{EV}$ in comparison to patients without EV. Even in the absence of a clear metabolic syndrome, both the degree of liver failure and BMI were independently associated with insulin resistance, suggesting a dual component of insulin resistance in cirrhosis (liver disease and overweight/obesity) ${ }^{[23]}$.

In our trial, we found that there were statistically significant increase in portal vein diameter (PVD), splenomegaly and lower platelet counts in patients with $\mathrm{EV}$ in comparison to patients without EV. Our findings were consistent with Cottone et al. ${ }^{[25]}$ who revealed that PVD, splenomegaly and low platelet count serve as predictors of EV.

In addition, our results showed statistically significant lower platelet count/spleen diameter ratio and higher right liver lobe diameter/albumin ratio in patients with $\mathrm{EV}$ in comparison to patients without $\mathrm{EV}$, which gave a strong relation with the development of EV and are considered good predictors for EV. Giannini et al ${ }^{[26]}$ reported that the use of the platelet count/spleen diameter ratio may be a tool to predict EV. This ratio links thrombocytopenia to splenomegaly to introduce a variable that takes into consideration that thrombocytopenia is mainly due to hypersplenism secondary to portal hypertension.

In our study, the cut-off value of the platelet count/spleen diameter ratio was lower than (1105.9) which is the optimal value for prediction of EV with a resulting $100 \%$ sensitivity and $98.8 \%$ specificity. Giannini $\boldsymbol{e t}$ $a^{a l} .^{[27]}$ reported the results of a multicenter study to validate the use of platelet count/spleen diameter ratio in the prediction of esophageal varices. At a cut-off value of 909, the sensitivity was $92 \%$ and the specificity was $67 \%$. In the study of Esmat et al..$^{[13]}$, a cut-off value of 1326.6 for the platelet count/spleen diameter ratio was used with a resulting $96.34 \%$ sensitivity and $83.33 \%$ specificity.

In our study, the cut-off value for the right liver lobe diameter/albumin concentration ratio (4.1) was the optimal value for prediction of EV with a resulting $93.8 \%$ sensitivity and $90 \%$ specificity which agrees with Esmat $\boldsymbol{e t}$ al.$^{[13]}$ when a cut-off value of 4.4 for the right liver lobe diameter/albumin concentration ratio was used, the sensitivity was $91.46 \%$ and the specificity was $77.78 \%$.

Alempijevic et al.$^{[28]}$ investigated the right liver lobe diameter/albumin concentration ratio as a noninvasive predictor of esophageal varices. At a cut-off value of 4.4, the sensitivity was $83.1 \%$ and the specificity was $73.9 \%$.

Our results agreed with Stranges et al. ${ }^{[29]}$ who found that the levels of ALT and AST were significantly higher in IR patients, which may reflect more severe inflammatory injury and the presence of steatosis.

Finally, we concluded that, type-2 DM is a risk factor for development of $\mathrm{EV}$ in $\mathrm{HCV}$ induced cirrhosis. Platelet count/spleen bipolar diameter ratio and right liver lobe diameter/albumin ratio in addition to insulin resistance measured by HOMA-IR may give a good prediction for the presence of esophageal varices. Chronic HCV infection has a strong relationship with the development of Insulin resistance (IR) in non-diabetic patients.

\section{References}

1. World Health Organization (WHO): Global surveillance and control of hepatitis C. Report of a WHO Consultation organized in collaboration with the Viral Hepatitis Prevention Board, Antwerp, Belgium. J Viral Hepat ., 6(1): 35- 47.

2. Nascimbeni F, Pias R, Bellentani S et al. (2013): From NAFLD in clinical practice to answer from guidelines. J. Hepatol .,59: 859-71.

3. Garcia-Tsao G, Sanyal AJ, Grace ND (2013): Prevention and Management of Gastroesophageal Varices and Variceal Hemorrhage in Cirrhosis. Hepatology ,46 (3): 922-938.

4. Chouhan A, Sushma T, Sanjay D et al. (2015) A study of correlation of esophageal varices in cirrhotic patients with portal hemodynamics with special references to portal vein diameter, portal vein velocity, congestion index, liver vascular index. J Evolut Med Dental Sci., 4: 59-66.

5. Cherian JV, Deepak N, Ponnusamy RP, Somasundaram A, Jayanthi V (2011): Noninvasive predictors of esophageal varices. Saudi J Gastroenterol., 64-68. 
Relationship between Type 2 Diabetes Mellitus and the occurrence of Gastroesophageal...

6. Abu El Makarem MA, Shatat ME, Shaker Y et al. (2011): Platelet count/bipolar spleen diameter ratio for the prediction of esophageal varices: The special Egyptian situation. Hepat Mon., 11(4): 278-284.

7. Berzigotti A, Seijo S, Reverter E et al. (2013): Assessing portal hypertension in liver diseases. Expert Rev. Gastroenterol. Hepatol.,7(2):141-155.

8. American Diabetes Association (2016): Standards of medical care in diabetes-2016: Summary of revisions. Diabetes Care, 39: S4-5.

9. Camma C, Petta S, Di Marco V et al. (2009): Insulin resistance is a risk factor for esophageal varices in hepatitis $\mathrm{C}$ virus cirrhosis. Hepatology ,49: 195-203.

10. Grundy SM, Cleeman JI, Daniels SR et al. (2015): Diagnosis and management of the metabolic syndrome: an American Heart Association/National Heart, Lung, and Blood Institute Scientific Statement. Circulation,112: 2735-52.

11. Li X, Wu K and Fan D (2009): Insulin resistance and platelet count/spleen diameter ratio: two simple, easy-to-get tests for predicting esophageal varices in cirrhosis. Hepatol., 49: 1394-1395.

12. Hung $\mathrm{CH}$, Lee $\mathrm{CM}$, Chen $\mathrm{CH}$ et al. (2009): Association of inflammatory and anti-inflammatory cytokines with insulin resistance in chronic hepatitis C. Hepatol., 47:110-126.

13. Esmat S, Omarn D and Rashid L (2012): Can we consider the right hepatic lobe size/albumin ratio a noninvasive predictor of esophageal varices in hepatitis $\mathrm{C}$ virus-related liver cirrhotic Egyptian patients? Eur JIntern Med.,23: 267-272.

14. Flegal K, Kit B, Graubard B et al. (2013): Association for all-cause mortality with overweight and obesity using standard body mass index categories: a systemic review and meta-analysis. JAMA., 309:71-82.

15. Matthews DR, Hosker JP, Rudenski AS et al. (1985): Homeostasis model assessment: insulin resistance and beta cell function from fasting plasma glucose and insulin concentrations in man. Diabetologia, 28:412-9.
16. Pugh RN, Murray-Lyon IM, Dawson JL et al. (1973): Transection of the esophagus for bleeding esophageal varices. Br J Surg., 60:646-649.

17. Alempijevic T, Bulat V, Djuranovic $S$ et al. (2014): Right liver lobe/albumin ratio: Contribution to non-invasive assessment of portal hypertension. World J Gastroenterol.,13(40): 53315335.

18. Castera L, Pinzani $M$ and Bosch $J$ (2012): Noninvasive evaluation of portal hypertension using transient elastography. J. Hepatol., 56: 696703.

19. Iwakiri Y and Groszmann RJ (2007): Vascular endothelial dysfunction in cirrhosis. J Hepatol., 46: 927-34.

20. Yosry A, Fouad R, Abdel Bary M, et al. (2009): Non-Invasive Prediction of Varices in Egyptian Cirrhotic Patients. Med. J. Cairo Univ; 77 (1): 343-349.

21. Shintani Y, Fujie H, Miyoshi $\mathrm{H}$ et al. (2004): Hepatitis $C$ virus infection and diabetes: direct involvement of the virus in the development of insulin resistance. Gastroenterol., 126(3): 840-848.

22. Antuna-Puente B, Disse $\mathbf{E}$ and RabasaLhoret $R$ (2011): How can we measure insulin sensitivity/resistance? Diabetes Metab., 37:179-188.

23. Erice E, Llop E, Berzigotti A, Abraldes JG, Conget I, Seijo S et al. (2012): Insulin resistance in patients with cirrhosis and portal hypertension. Am J Physiol Gastrointest Liver Physiol., 302: G1458-G1465.

24. Wasfy E, Elkassas G, Elnawasany S, Elkasrawy K et al. (2018): Predicting esophageal varices in cirrhotic hepatitis $\mathrm{C}$ virus patients using noninvasive measurement of insulin resistance variables. Endocr Metab Immune Disord Drug Targets, 18(6): 573-580.

25. Cottone M, D'Amico G, Maringhini A et al. (2009): Predictive Value of Ultrasonography in the Screening of Non-Ascitic Cirrhotic Patients with Large Varices. J Ultrasound Med., 5:189-192.

26. Giannini E, Botta F, Borro P, Risso D, Romagnoli $P$ and Fasoli A (2003): Platelet count/spleen diameter ratio: 
proposal and validation of a noninvasive parameter to predict the presence of esophageal varices in patients with liver cirrhosis. Gut, 52:1200-1205.

27. Giannini EG, Zaman A, Kreil A et al. (2006): Platelet count/spleen diameter ratio for the noninvasive diagnosis of esophageal varices: results of a multicenter, prospective, validation study. Am J Gastroenterol., 101: 2511-2519.
28. Alempijevic T, Bulat V, Djuranovic S, Jesic $R$ et al. (2007): Right liver lobe/albumin ratio: contribution to non-invasive assessment of portal hypertension. World J Gastroenterol., 13:5331-5.

29. Stranges S, Trevisan M, Dorn JM et al. (2005): Body distribution, liver enzymes, and risk of hypertension: evidence from the Western New York Study. Hypertension,46(5):1186-93. 